\title{
Masers in Starburst Galaxies
}

\author{
Jeremy Darling \\ Department of Astrophysical and Planetary Sciences \\ University of Colorado \\ Boulder, CO 80309, United States of America \\ email: jdarling@colorado.edu
}

\begin{abstract}
Masers in starburst galaxies are outstanding probes of a range of phenomena related to galaxy and black hole evolution, star formation, and magnetic fields. Here I briefly discuss five related topics: (1) Galactic analog water masers in nearby galaxies; (2) multiwavelength solutions to the $\mathrm{OH}$ megamaser puzzle in major galaxy mergers; (3) formaldehyde anti-inversion in starburst galaxies; (4) $\mathrm{OH}$ spoofing in HI surveys; and (5) new discovery space in radio line surveys. New insights into the physical conditions responsible for $\mathrm{OH}$ megamasers, including indications of a critical molecular gas density obtained from the formaldehyde "densitometer," will be applicable to future surveys, particularly surveys for redshifted hydrogen where OH lines arising in major galaxy mergers can "contaminate" the disk population identified by the HI 21 $\mathrm{cm}$ line. Blind radio spectral line surveys also offer the opportunity for unexpected discoveries of new nonthermal radio lines.
\end{abstract}

Keywords. masers, radiation mechanisms: nonthermal, radiative transfer, surveys, galaxies: evolution, galaxies: interactions, galaxies: ISM, galaxies: starburst, infrared: galaxies, radio lines: galaxies

\section{Introduction}

The state of observations of extragalactic masers is woefully inadequate, particularly compared to the wealth of Galactic maser data. It is important to remember that all masers seen in the Galaxy occur in other galaxies and that there are masers produced in other galaxies that are not seen in our own. The latter group include both the well-known water and hydroxyl megamasers as well as masers yet to be discovered (see Section 6).

The reason for the paucity of information about Galactic analog masers in other galaxies lies in the raw sensitivity needed to detect masers at Mpc distances. For example, moving from kpc to Mpc distances, resolution drops 1000-fold, and luminosity sensitivity is effectively diminished million-fold: parsecs become $\mathrm{kpc}$, and $1 \mathrm{Jy}$ becomes $1 \mu \mathrm{Jy}$. There are thus only a few maser species detected in external galaxies, most often water and hydroxyl, and they are generally not Galactic analogs.

Here I present a brief discussion of five related topics: (1) Galactic analog water masers in nearby galaxies; (2) solutions to the $\mathrm{OH}$ megamaser puzzle in major galaxy mergers; (3) formaldehyde anti-inversion in starburst galaxies; (4) OH spoofing in HI surveys; and (5) new discovery space in radio line surveys.

\section{Water Masers in Star-Forming Galaxies}

Despite the difficulty of detecting Galactic analog masers in galaxies beyond the Magellanic Clouds, modern telescopic facilities now have the raw sensitivity to detect Galactic analog water masers to distances of $\sim 20 \mathrm{Mpc}$. If we call a Galactic analog maser one that simply has a peak flux density or isotropic line luminosity equal to or less than the brightest Galactic masers, then we now have numerous examples of such extragalactic 

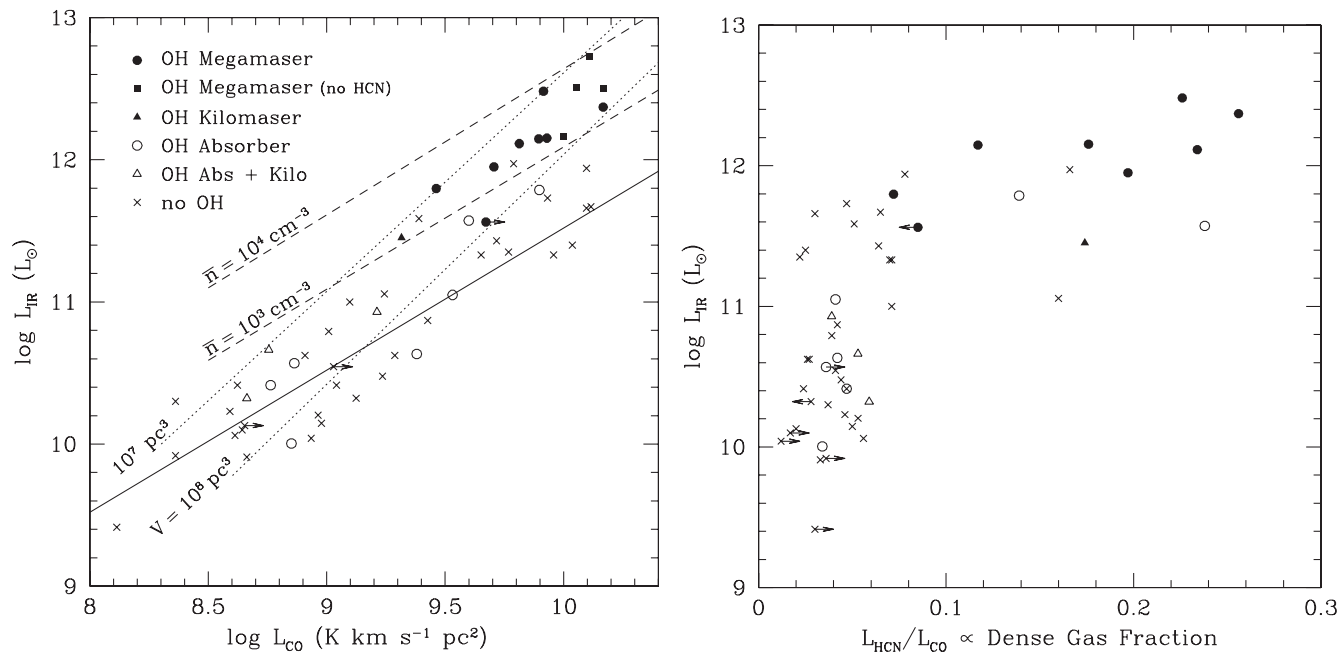

Figure 1. Global star formation properties of $\mathrm{OH}$ megamaser host galaxies (after Darling 2007). Left: IR luminosity versus CO line luminosity in HCN-detected galaxies with known $\mathrm{OH}$ properties from the Gao \& Solomon (2004a) sample. The legend indicates symbols for $\mathrm{OH}$ megamasers, $\mathrm{OH}$ kilomasers, $\mathrm{OH}$ absorbers, and objects with no detected $\mathrm{OH}$ lines. The solid line is a linear fit by Gao \& Solomon (2004b) to galaxies with $L_{\mathrm{IR}}<10^{11} L_{\odot}\left(L_{\mathrm{IR}}=33 L_{\mathrm{CO}}\right.$ in the units above). The dotted lines indicate a constant total volume of molecular material, and the dashed lines indicate the mean $\mathrm{H}_{2}$ density derived from Krumholz \& Thompson (2007). Right: IR luminosity versus $L_{\mathrm{HCN}} / L_{\mathrm{CO}}$, a proxy for the dense gas fraction. $\mathrm{OH}$ megamaser hosts form the majority of the high density population, suggesting an extreme stage of star formation and tidal concentration of the ISM.

masers (e.g., Castangia et al. 2008, Darling et al. 2008, Surcis et al. 2009, Tarchi et al. 2011). But in most cases the physical conditions, such as those found in dwarf starbursts or the Antennae Galaxies (a major merger), are not analogous to those found in the Galaxy (e.g., Brogan et al. 2010).

There have, however, recently been detections of true Galactic analog masers in the Andromeda Galaxy, M31: Sjouwerman et al. (2010) detected a $6.7 \mathrm{GHz}$ methanol maser, and Darling (2011) detected five water maser complexes. All of these masers appear to be associated with star formation and lie within the molecular gas- and dust-rich part of the spiral disk of M31.

\section{The OH Megamaser Puzzle}

Lo (2005) posed a useful question about the hosts of $\mathrm{OH}$ megamasers that can be paraphrased as: suppose that one can identify two apparently identical major mergers/(U)LIRGs, but one shows OH megamaser (OHM) emission while the other does not. What is the cause of the OHM? What is physically different between these galaxies? It may be that one cannot find such a situation, but more broadly, it is the case that the global properties, such as IR luminosity, IR color, morphology, and optical spectrum do not distinguish between OHM hosts and non-masing mergers once the selection for the proper type of galaxy (e.g., late-stage merger, $L_{\mathrm{IR}} \gtrsim 10^{11.6} L_{\odot}$, warm IR colors, starburst-dominated spectrum) has been made. Could the difference be beaming? Small scale physical conditions? OH abundance? AGN influence? Or could it be some property not yet observed? 

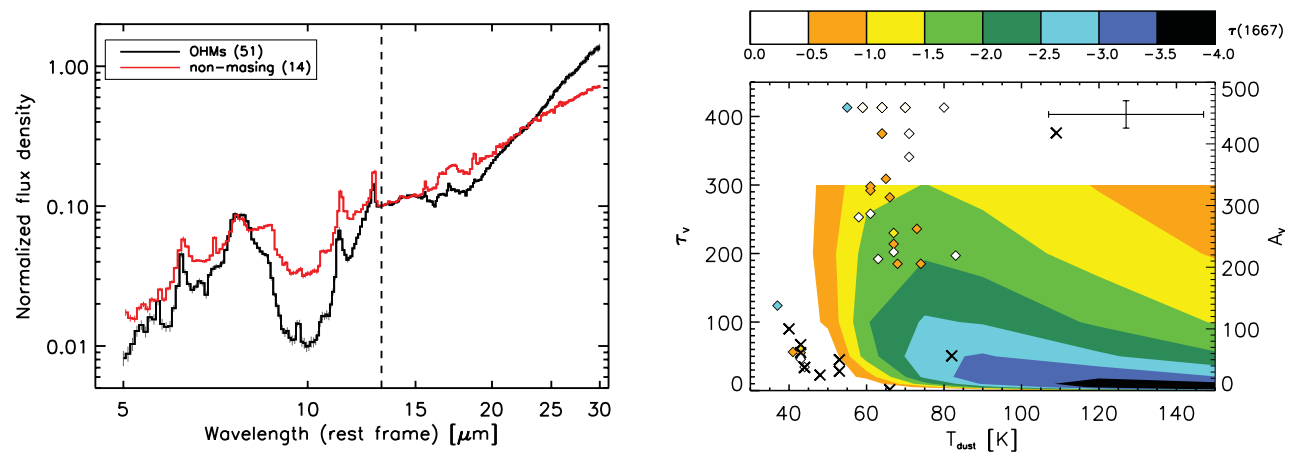

Figure 2. Comparison of IR properties of $\mathrm{OH}$ megamaser host galaxies to non-masing but otherwise similar galaxies (after Willett et al. 2011b). Left: The average mid-IR Spitzer spectrum of OHM hosts shows a deeper $10 \mu \mathrm{m}$ silicate dust absorption feature as well as a steeper 20-30 $\mu \mathrm{m}$ dust slope when compared to non-masing galaxies with similar global properties. These differences are two sides of the same coin: a very geometrically and optically thick dust blanket $\left(\tau_{V} \sim 300\right)$ in OHM hosts (Ivezić \& Elitzur 1997, Levenson et al. 2007). Right: Comparison of the observed dust optical depth versus dust temperature to the theoretical predictions of Lockett \& Elitzur (2008). Colors in contours indicate the predicted $1667 \mathrm{MHz} \mathrm{OH}$ line optical depth (negative indicates masing), and colors in the points indicate the observed apparent $1667 \mathrm{MHz}$ $\mathrm{OH}$ line optical depth. Crosses indicate non-masing galaxies. While the OHM sample is well-separated from the non-masing sample in this space, and we can now predict the most likely galaxies to produce OHM activity, the theory cannot yet predict the observed maser emission based on global dust properties alone.

The root physical cause of $\mathrm{OH}$ megamaser activity has been observationally revealed to lie in the extreme concentration of gas and dust in major mergers, and OHMs appear to select the time of peak concentration during the merging process.

Darling (2007) show that OHMs lie in a distinct locus in plots of star formation rate (as traced by IR luminosity) versus CO line luminosity: the OHM hosts are the starburst galaxies that cause the IR-CO relation to break away from the linear relationship that holds for star-forming galaxies across many orders of magnitude (Gao \& Solomon 2004b). This suggests that OHM hosts are undergoing a special stage of merger-triggered star formation. OH "kilomasers" and absorbers can be found throughout the linear part of the relation, suggesting that $\mathrm{OH}$ abundance is not a factor in producing OHMs (Figure 1). OHM hosts are also the majority of galaxies in the high dense gas fraction phase of the merging process, suggesting that while OHM emission is beamed, it is stochastic and fairly isotropic when conditions are favorable for OHM production.

Willett et al. (2011a, 2011b) show that OHM hosts have a deeper $10 \mu \mathrm{m}$ silicate dust absorption feature and steeper $20-30 \mu \mathrm{m}$ dust continuum slope than non-masing but otherwise similar galaxies (Figure 2). Ivezić \& Elitzur (1997) demonstrate that for silicate dust, these are two aspects of the phenomenon: as dust opacity increases, the mid-IR silicate dust absorption features become so deep that they steepen the mid-IR slope of the dust emission and move the peak to longer wavelengths. In fact, for $\tau_{V} \sim 300$, the peak of the dust emission is shifted to maximize the IR pumping at 35 and $53 \mu \mathrm{m}$ that is most likely responsible for OHM emission.

Willett et al. (2011b) directly compare observations of the OHM and non-masing galaxy host dust temperatures and apparent dust optical depths to the locus theoretically predicted by Lockett \& Elitzur (2008) for OHM production and find a significant separation between $\mathrm{OHM}$ hosts and non-masing galaxies, verifying that non-masing galaxies do not lie in the expected dust opacity-temperature region required for masing. Willett 
et al. (2011b) also demonstrate that the theoretical dust optical depths may be lower than what is observed (Figure 2).

Finally, in the Mangum et al. (2008) study of formaldehyde $\left(\mathrm{H}_{2} \mathrm{CO}\right)$ in nearby starforming galaxies, $\mathrm{OHM}$ hosts show the $6 \mathrm{~cm} \mathrm{H}_{2} \mathrm{CO}$ line in emission while all non-masing host galaxies show this line in absorption. This line sign-flip occurs when the $6 \mathrm{~cm}$ line excitation temperature crosses the cosmic microwave background (CMB) temperature, which corresponds to a molecular hydrogen density threshold of $n\left(\mathrm{H}_{2}\right) \sim 10^{5.6} \mathrm{~cm}^{-3}$ (the $\mathrm{H}_{2} \mathrm{CO} \mathrm{cm}$ lines are collisionally driven to low excitation temperatures; see Section 4). Again, this suggests a density threshold above which OHM activity can (or perhaps must) occur.

The pieces of the OHM puzzle are falling into place and are in good agreement: we have a picture of a stage during major galaxy mergers when there is extreme concentration of gas and dust in a period of peak triggered star formation. The combination of extreme concentration of a large fraction of a galaxy's ISM into the inner kpc with warm and geometrically and optically thick dust driven to peak emission near the OHM pumping lines produces the conditions for OHM production in the inner $\sim 100 \mathrm{pc}$. These conditions are physically wide-spread and stochastic, producing many masing sightlines, making the OHM detectable from most viewing angles. This also explains why OHM emission is predictable based on global gas and dust properties of the host galaxies rather than being an amplification of small-scale conditions.

\section{Formaldehyde Anti-Inversion}

Centimeter-wave $K$-doublet rotational transitions of formaldehyde $\left(\mathrm{H}_{2} \mathrm{CO}\right)$ can be collisionally "refrigerated" below the cosmic microwave background (CMB) temperature such that these transitions absorb CMB photons (Palmer et al. 1969, Townes \& Cheung (1969), Evans et al. 1975, Garrison et al. 1975). The manner in which the cm lines of $\mathrm{H}_{2} \mathrm{CO}$ are cooled is analogous to a maser pumping process: a pump drives an overpopulation of states, but in this case they are lower-energy states, and the level populations become "anti-inverted" compared to thermal. When the line excitation temperature drops below the local CMB temperature, CMB photons may be absorbed. This effect of "anomalous" $\mathrm{H}_{2} \mathrm{CO}$ absorption was playfully called the dasar: darkness amplification by stimulated absorption of radiation (an inaccurate description since the "darkness" is in fact a $2.73 \mathrm{~K}$ blackbody and the absorption is not stimulated).

Since absorption lines are detectable independent of distance, and since the CMB illuminates all gas and provides an exceptionally uniform illumination that lies behind every galaxy, a Formaldehyde Deep Field can provide a mass-limited census of the dense molecular gas associated with star formation. If multiple lines are observed then physical molecular gas densities may also be obtained fairly independently of the gas kinetic temperature or other factors. The utility of the "formaldehyde densitometer" has been well-studied in the Galaxy (e.g., Mangum \& Wootten 1993, Ginsburg et al. 2011), in nearby star-forming galaxies (e.g., Mangum et al. 2008), in the $z=0.67$ gravitational lens and molecular absorption line system (Zeiger \& Darling 2010), and theoretically for arbitrary redshift (Darling \& Zeiger 2012, submitted).

\section{OH Spoofing HI}

Briggs (1998) predicted that $18 \mathrm{~cm} \mathrm{OH}$ megamaser lines produced in major galaxy mergers will "contaminate" blind HI $21 \mathrm{~cm}$ surveys (which typically identify spiral disks), and the level of contamination will depend on the sensitivity and redshift coverage of 
the survey. In fact, for any given survey depth, there is a point in redshift space where the sky density of the "contaminating" lines surpasses the sky density of the survey object line and becomes the dominant population. This is due to the relatively fixed HI masses of massive disk galaxies (fixed to within one order of magnitude, even for early gas-rich galaxies) compared to the highly nonlinear maser action that can make lines disproportionately bright compared to the gas mass responsible for the maser emission.

The expected sky density of disks versus mergers, for a given survey sensitivity and frequency span (redshift range), depends primarily on the merging rate of galaxies as a function of cosmic time and on the OH luminosity function (Briggs 1998, Darling \& Giovanelli 2002). The galaxy merging rate versus redshift is a topic of ongoing contention and is highly selection-biased. The $\mathrm{OH}$ luminosity function, while fairly well-measured given the small sample size, is not well-defined at the high luminosity end, which is precisely the luminosity that future surveys will detect first given the large volumes that will be sampled and the severe Malmquist bias of OHM samples in general.

To address these issues, we have begun a multi-wavelength data-mining approach to sifting HI survey samples for $\mathrm{OH}$ contamination using the Arecibo Legacy Fast Arecibo L-band Feed Array (ALFALFA) survey (Giovanelli et al. 2005) as a test case, training set, and proof of concept. While mergers are IR-luminous, lower redshift star-forming disk galaxies can show a similar flux density in the mid-IR bands, so IR brightness alone is an insufficient selection condition. However, lack of a bright IR counterpart can exclude $\mathrm{OH}$, thus confirming $\mathrm{HI}$ as the line identification. We also employ optical counterpart identification (which is not always unique), optical morphology, mid-IR color, photometric redshift, and radio line profile to sift $\mathrm{OH}$ from $\mathrm{HI}$ signals. None of these criteria alone are sufficient, but taken together they can usually distinguish disks at $z=0-0.05$ from mergers at $z=0.17-0.23$. In some cases, however, the only certain discrimination comes from an optical spectroscopic redshift.

We anticipate that this process of sorting disks from mergers will be challenging in redshifted HI surveys planned for Square Kilometer Array prototypes such as MeerKAT, ASKAP, SKAMP, FAST, and Apertif on the WSRT, both due to noisy spatially unresolved signals toward faint optical/IR counterparts and due to the higher "contamination" rate expected in these surveys compared to ALFALFA. It is also likely that new lines will be detected that are neither $\mathrm{HI}$ nor $\mathrm{OH}$, making the sifting process even more challenging.

\section{New Discovery Space}

Low energy (frequency) transitions are slow: the Einstein coefficient for spontaneous emission scales as $A \propto \nu^{3}$, so metastable states are more common at low energies. Many low-energy transitions connect energy levels split by hyperfine structure or lambdadoubling, and so are also forbidden. Moreover, slight imbalances in rates of higher energy (fast) transitions can produce large nonthermal populations in low energy states, driving inversion (or anti-inversion). Note that the Boltzmann factors for low-energy states are generally roughly unity: $1-\epsilon$ thanks to the $\mathrm{CMB} T_{\mathrm{CMB}}=(1+z) \times 2.73 \mathrm{~K}$ radiation field $\left(k T_{\mathrm{CMB}} \gg h \nu\right)$.

Pumping cycles for masers are not intuitive and rely on the detailed structure of the many states and rates connected (and even not connected) to the states involved in the maser. It is difficult to explain - much less predict - maser (or dasar) activity. Examples include Ly $\alpha$ pumping of the $21 \mathrm{~cm}$ HI line, which is fairly straightforward, to $\mathrm{H}_{2}$ collisional pumping of $\mathrm{H}_{2} \mathrm{CO}$, to IR radiative pumping of $\mathrm{OH}$. Note that the latter two examples were not predicted before they were observed. 
The point of this discussion is that we simply do not know what bright maser or other nonthermal lines will appear at low frequencies. It is likely that new masers will be discovered in the course of redshifted HI $21 \mathrm{~cm}$ line surveys with the Square Kilometer Array and its prototypes. Extensive and sensitive sub-GHz spectral surveys have not been pursued due in large part to RFI, but also due to lack of community interest. It is difficult to propose to "make discoveries" when one does not know what one is looking for or might find; no one wrote a proposal to detect "mysterium," the first astrophysical maser.

\section{Conclusions}

Current telescopic facilities now have the raw sensitivity to detect Galactic analog masers in other galaxies. Using Galactic analog masers as extragalactic probes will be a growing field of astrophysical inquiry.

In contrast, the exceptional $\mathrm{OH}$ megamasers detected in starbursts have no local analogs and have been a long-term puzzle, but by using multi-wavelength observations combined with theoretical models we now have a better notion of the physical conditions and host galaxies indicated by the bright $\mathrm{OH}$ megamaser signposts. With this new understanding, we can interpret the populations of mergers and exceptional starbursts that "contaminate" HI $21 \mathrm{~cm}$ surveys via OH megamaser "spoofing." We can also learn how to separate disks from mergers in blind high-redshift HI $21 \mathrm{~cm}$ surveys planned for Square Kilometer Array prototypes.

Anti-inverted formaldehyde lines provide a molecular gas "densitometer" and the absorption of $\mathrm{CMB}$ photons by $\mathrm{H}_{2} \mathrm{CO}$ provides a probe of star formation at any redshift. We envision a Formaldehyde Deep Field that will make a dust extinction-free redshiftindependent molecular gas mass-limited census of the history of cosmic star formation (Darling \& Zeiger 2012, submitted).

Finally, it is likely that new nonthermal lines will be discovered in low frequency radio spectral surveys provided that observers don't pre-bias surveys and data pipelines to only detect what is already expected and known.

\section{References}

Briggs, F. H. 1998, A\&A, 336, 815

Brogan, C., Johnson, K., \& Darling, J. 2010, ApJ, 716, L51

Castangia, P., Tarchi, A., Henkel, C., \& Menten, K. M. 2008, A\& A, 479, 111

Darling, J. \& Giovanelli, R. 2002, ApJ 572, 810

Darling, J. \& Giovanelli, R. 2006, $A J$ 132, 2596

Darling, J. 2007, ApJ, 669, L9

Darling, J., Brogan, C., \& Johnson, K. ApJ, 685, L39

Darling, J. 2011, ApJ, 732, L2

Evans, N. J. II, Zuckerman, B., Morris, G., \& Sato, T. 1975, ApJ, 196, 433

Gao, Y. \& Solomon, P. M. 2004a, ApJS, 152, 63

Gao, Y. \& Solomon, P. M. 2004b, ApJ 606, 271

Garrison, B. J., Lester Jr., W. A., Miller, W. H., \& Green, S. 1975, ApJ, 200, L175

Ginsburg, A., Darling, J., Battersby, C., Zeiger, B., \& Bally, J. 2011, ApJ, 736, 149

Giovanelli, R., Haynes, M. P., Kent, B. R., et al. 2005, AJ, 130, 2598

Henkel, C., Walmsley, C. M., \& Wilson, T. L. 1980, A\& A, 82, 41

Ivezić, Ẑ. \& Elitzur, M. 1997, MNRAS, 287, 799

Jethava, N., Henkel, C., Menten, K. M., Carilli, C. L., Reid, M. J., \& Walmsley, C. M. 2007, $A \& A, 472,435$ 
Krumholz, M. R. \& Thompson, T. A. 2007, ApJ, 669, 289

Levenson, N. A., Sirocky, M. M., Hao, L., Spoon, H. W. W., Marshall, J. A., Elitzur, M., \& Houck, J. R. 2007, ApJ, 654, L45

Lo, K. Y. 2005, ARAA 43, 625

Lockett, P. \& Elitzur, M. 2008, ApJ, 677, 985

Mangum, J. G. \& Wootten, A. 1993, ApJS, 89, 123

Mangum, J. G., Darling, J., Menten, K. M., \& Henkel, C. 2008, ApJ, 673, 832

Palmer, P., Zuckerman, B., Buhl, D., \& Snyder, L. E. 1969, ApJ, 156, L147

Sjouwerman, L. O., Murray, C. E., Pihlstrom, Y. M., Fish, V. L., \& Araya, E. D. 2010, ApJ, 724, L158

Surcis, G., Tarchi, A., Henkel, C., Ott, J., Lovell, J., \& Castangia, P. 2009, A $\mathscr{J} A, 502,529$

Tarchi, A., Castangia, P., Henkel, C., Surcis, G., \& Menten, K. M. 2011, A\& A, 525, A91

Townes, C. H. \& Cheung, A. C. 1969, ApJ, 157, L103

Willett, K. W., Darling, J., Spoon, H. W. W., Charmandaris, V., \& Armus, L. 2011, ApJS, 193, 18

Willett, K. W., Darling, J., Spoon, H. W. W., Charmandaris, V., \& Armus, L. 2011, ApJ, 730, 56

Zeiger, B. \& Darling, J. 2010, ApJ, 709, 386 